\title{
Uso de Analytics no Apoio da Tomada de Decisões e na Eficiência Operacional do Hospital Santa Izabel
}

\author{
Karla Aleluia ${ }^{1}$ \\ ${ }^{1}$ Gerência de Tecnologia da Santa Casa de Misericórdia da Bahia; Salvador, Bahia, Brasil

\section{Introdução}

As Unidades de Negócio que compõem a estrutura da Santa Casa da Bahia (SCBA) geram um conteúdo massivo e diverso de dados, através dos inúmeros processos já mapeados em seus sistemas de gestão e aplicações integradas, que por sua vez são alimentados por pessoas ou através de sensores, dispositivos e plataformas digitais. Diante desta evidência, questiona-se:

\section{Qual o valor que tais dados gerados nessa cadeia podem trazer para os negócios da Instituição?}

Correspondence addresses: Karla Aleluia

karla.aleluia@santacasaba.org. br

Received: July 17, 2019

Revised: July 31, 2019

Accepted: August 13, 2019

Published: September 27, 2019

Data Availability Statement: All relevant data are within the paper and its Supporting Information files.

Funding: This work was the result of author's initiative. There was no support of research or publication funds.

Competing interests: The author has declared that no competing interests exist.

\section{Copyright}

(C) 2019 by Santa Casa de Misericórdia da Bahia. All rights reserved. ISSN: 2526-5563
Ações que abordam Pessoas, Processos e Tecnologia podem ser observadas através de diversas iniciativas estratégicas da SCBA, que englobam a sinergia entre tais elementos para garantir os resultados esperados. Em particular, podemos citar as ações do Hospital Santa Izabel, vinculadas a iniciativas bem estabelecidas e apoiadas por metodologias consolidadas de melhorias dos processos em saúde.

Diante dessas considerações, uma nova abordagem tem sido requerida à medida em que o dado se torna um ativo essencial no funcionamento de iniciativas estratégicas. A Figura 1, extraída de pesquisas do Gartner, ilustra que o dado se destaca como um novo elemento para dar suporte ao gerenciamento das iniciativas organizacionais. Dados estruturados e não estruturados, das mais variadas fontes, que devidamente capturados são volumosos, têm se tornado ferramentas para tomada de decisões. Além de apoiar decisões estratégicas, os dados passam a ter papel fundamental para o desenvolvimento dos negócios, em particular quando pensamos no segmento de saúde.

Diante desse contexto, a necessidade de uma nova habilidade estratégica se destaca como primordial no avanço das iniciativas orientadas ao uso de dados e informações, que é a capacidade analítica, também descrita na literatura como Data Literacy. Cuidar do dado passou a ser, portanto, ponto crítico para que se possa garantir a sinergia do dado como novo elemento, introduzido com a evolução da sociedade digital.

Esse artigo tem por objetivo apresentaras iniciativas analíticas, denominada de Analytics, desenvolvidas para o projeto estratégico de eficiência 
Figura 1. Evolução da tríplice Pessoas, Processos e Tecnologia, segundo o Gartner.

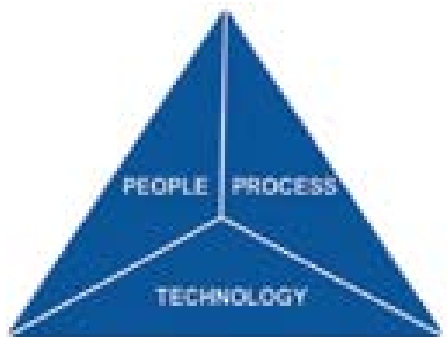

operacional do Hospital Santa Izabel através do monitoramento do resultado assistencial do paciente, e demonstrar a importância da cadeia de valor do dado para uma gestão mais eficiente e assertiva.

\section{A Cadeia de Valor do Dado}

Esta seção apresenta, em uma visão prática, o comportamento da cadeia de valor do dado e seu processo de transformação.

É comum usarmos os termos "dado" e "informação" como sinônimos. Entretanto, a evolução da cadeia dado - informação conhecimento - sabedoria é o que diferencia o uso de tais terminologias.

Dado é uma sequência de símbolos quantificados, enquanto que informação é uma abstração ou uma interpretação baseada em um contexto.

Transformar dado em informação significa contextualizar símbolos quantificados. O conhecimento é alcançado através da experimentação pessoal.

Tal conceito pode ser exemplificado da seguinte forma: 80 é uma sequência de símbolos ou caracteres, portanto, é um dado. Tal sequência em uma lista de ocupação hospitalar sugere a informação de 80 unidades de uma ocupação hospitalar. Um funcionário do hospital entende, por experiência, que se trata de um percentual de $80 \%$ de taxa ocupação atribuído a uma unidade de internação em um determinado período. Finalmente, um gestor tem a competência ou sabedoria para atuar sobre esse conhecimento e tomar uma decisão.
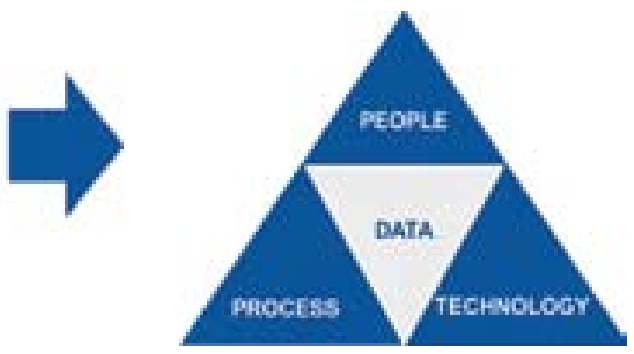

Apoiado no entendimento da evolução da cadeia de valor do dado e da capacidade analítica cada vez mais necessária dos profissionais que utilizam o dado como matéria-prima para a realização de suas atividades, observa-se que organizar e potencializar o uso dos dados é fundamental para a garantia do seu ciclo de vida.

Para o projeto de eficiência operacional do HSI, evidencia-se tal necessidade em diversos tipos de dados analisados, tais como as etapas dos tempos cirúrgicos. Ações em curso estão sendo tomadas para garantir um registro mais real e automático dos tempos que compõem o uso da sala cirúrgica, considerando que esse tempo é utilizado na cadeia de valor do dado para definir valores de custo e, consequentemente, do resultado operacional.

\section{O que é Analytics?}

O Gartner define Analytics como a "disciplina que aplica lógica e matemática aos dados para fornecer insights para tomar decisões". A partir desse conceito, passouse a construir uma cultura analítica à medida em que os dados passam a ser medidos e classificados para tomada de decisões.

Uma vez que os principais indicadores de desempenho são identificados e comparados com suas respectivas metas, somos capazes de monitorar os valores reais em relação aos objetivos estratégicos, bem como realizar análises históricas e até mesmo tendências futuras. Esta é a capacidade de medir. 
Tais informações podem ser combinadas com diferentes fontes de dados para que cheguem a um nível maior de detalhe, chamado também de menor granularidade. A partir daí, é possível identificar os atributos mais importantes para tal nível de detalhe, encontrar associações ou cluster de dados, ou até mesmo construir grupos personalizados de dados. Ao evoluir com essas características, alcança-se a capacidade de classificar.

Com a posse de informações medidas e classificadas, é possível construir mais transparência na tomada de decisão clínica, operacional ou estratégica; introduzir regras para decisões automatizadas e simular dados com base em tendências. Neste momento percebe-se a capacidade institucional de decidir.

Assim, considerando a aplicação prática, entende-se Analytics como a capacidade do Hospital Santa Izabel em medir, classificar e decidir, usando ferramentas que facilitam a análise e a apresentação das informações, construídas por equipe multidisciplinar, mesclando conhecimentos específicos do negócio, engenharia e ciência de dados.

Diferentes tipos de análises podem ser aplicados, a saber:

Análise Descritiva: essa análise traz uma fotografia do presente, mas também considera histórico de dados. O objetivo é gerar um panorama claro e preciso de um determinado assunto no presente momento, identificando o que aconteceu. Como exemplo, a análise descritiva responde a perguntas do tipo: quantos procedimentos cirúrgicos de Retirada de Duplo J foram realizados no ano de 2018 ?

Análise Diagnóstica: esse modelo de análise possibilita identificar causas e consequências percebidas ao longo do tempo, cruzando informações diversas, com o objetivo de entender quais fatores impactaram o resultado encontrado. Tais análises são úteis também para entender os fatores que precisam ser ajustados a fim de obter o resultado desejado. A análise diagnóstica responde, seguindo o exemplo anterior, o porquê do alcance do volume de procedimentos de Retirada de Duplo J realizados.

Análise Preditiva: análises preditivas abordam a questão "o que é provável que aconteça". Ela se baseia em técnicas como modelagem preditiva, análise de regressão, previsão, estatística multivariada e correspondência de padrões Machine Learning (ML) e Inteligência Artificial (IA). A probabilidade de aumentarmos o resultado operacional com a execução do procedimento de Retirada de Duplo J em uma unidade de Day Hospital é uma questão que pode ser aplicada nesse tipo de análise.

Análise Prescritiva: baseia-se em técnicas como análise de grafos, simulação, processamento de eventos complexos e mecanismos de recomendação para abordar questões do tipo "O que deve ser feito" ou "O que podemos fazer para algo acontecer".

\section{Do Business Intelligence (BI) para o Analytics}

Desde a migração do Sistema Hospitalar Corporativo da Santa Casa da Bahia para a solução da MV, em 2016, e das iniciativas de transformação digital requeridas pelas certificações hospitalares, tais como Qmentum e HIMSS, observa-se um avanço na geração de uma diversidade de dados cada vez mais complexa. À medida que os diversos contextos e processos em saúde são mapeados no MV e nas aplicações integradas, o volume de dados gerados como resultados de tais automatizações é ampliado. Dados que, assim como os sistemas, precisam estar integrados e associados aos diversos contextos e visões de processos. Para o monitoramento de tais 
processos e análise dos dados gerados, foi criada na SCBA, através de equipe pertencente à Gerência de Tecnologia da Informação e Comunicação (GTI), uma estrutura de Business Intelligence (BI), em que diversos assuntos foram mapeados em um ambiente específico, com banco de dados modelados em Data Warehouse (DW), dashboards e relatórios. Esse complexo ambiente foi chamado de Portal da Informação, em concordância com o framework apresentado na Figura 2.

O Portal da Informação da SCBA representa o primeiro domínio deste framework e é composto por três camadas, na qual a primeira camada representa as fontes de dados e seus processos de transformação, a segunda camada representa os assuntos do DW e os modelos estatísticos e, por fim, a última camada, ou camada de apresentação, disponibiliza os dashboards no Portal Corporativo, conforme ilustra a Figura 3.

Com as iniciativas do projeto de eficiência operacional para monitoramento do resultado assistencial, surgiu anecessidade de evoluirmos o Portal da Informação para o domínio da bancada analítica (Analytics Workbench),

Figura 2. Quatro domínios de Analytics, segundo o Gartner.

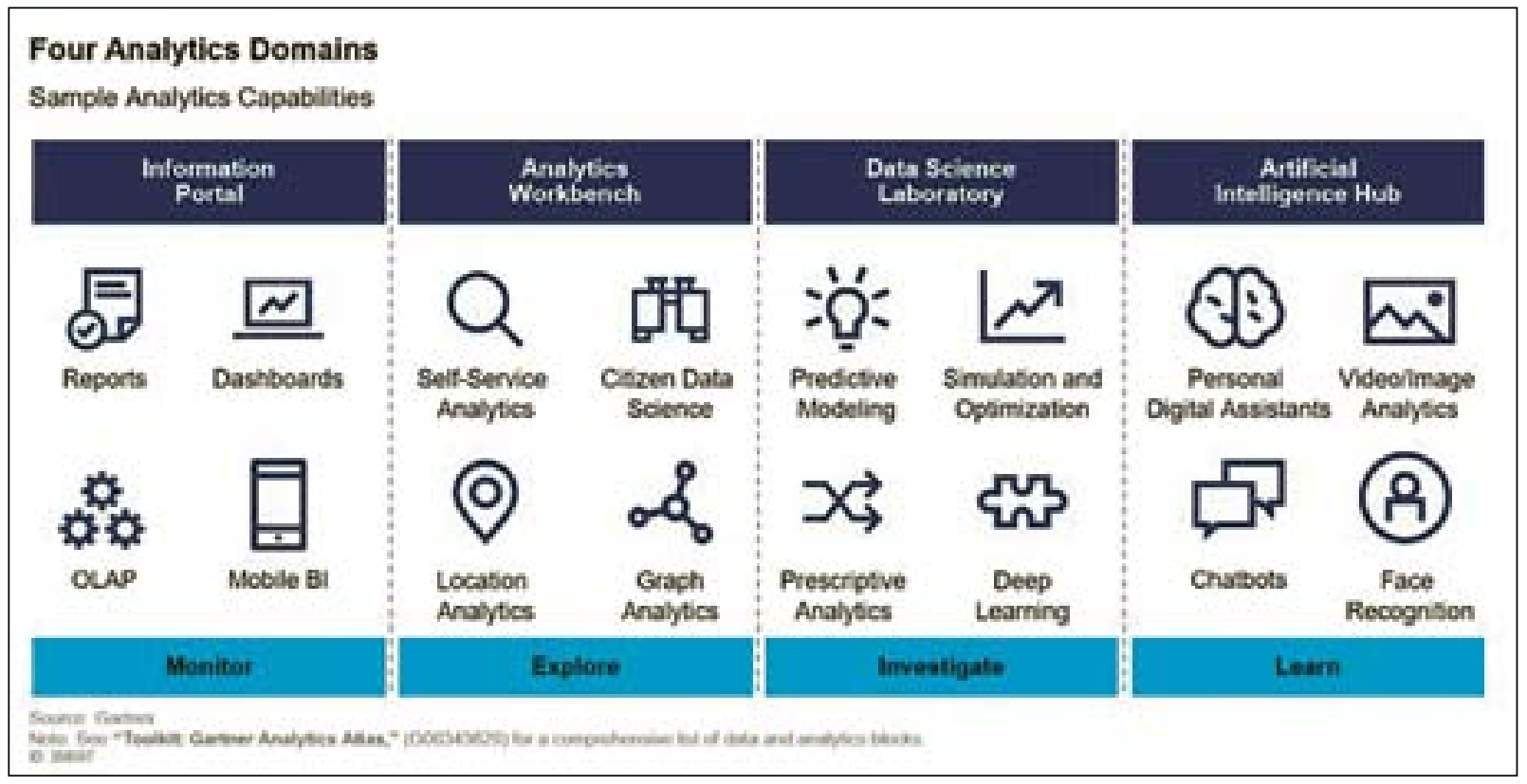

Figura 3. Três camadas do Portal da Informação da Santa Casa da Bahia.

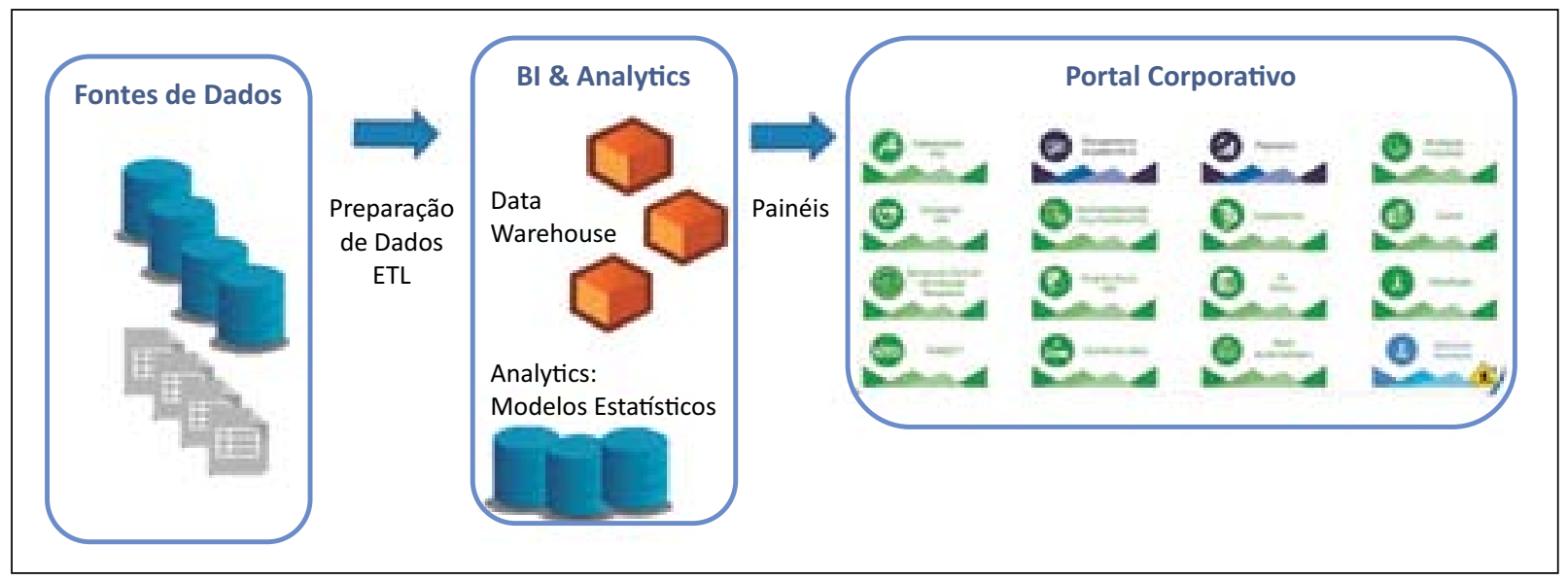


ilustrado na Figura 2, com o objetivo de ampliar as capacidades de exploração dos dados e tipos de análises. Ferramentas de visualização e SelfService $B I$ estão sendo avaliadas para compor esse ambiente, permitindo tal ampliação na exploração e até mesmo na investigação com modelagem preditiva.

\section{Analytics para apoiar o Projeto Eficiência Operacional com o Uso de Inteligência Artificial (IA).}

O objetivo inicial deste projeto liderado pela alta gestão do Hospital Santa Izabel foi buscar dados que compõem o resultado dos procedimentos hospitalares de internação para análise do resultado assistencial dos pacientes.

A construção de uma solução de Analytics para apoiar este projeto foi realizada em algumas etapas.

Etapa 1: Desenvolvimento do DW e Preparação dos Dados

Nessa primeira etapa foi fundamental identificar os dados necessários para se chegar ao resultado assistencial. Para tanto, visões de custo e de receita foram relacionadas de forma a trazer dados íntegros em uma mesma granularidade. O maior desafio foi buscar relacionamentos confiáveis e consistentes entre dados de fontes diversas, tais como custos, receita e atendimento do paciente. Diversas métricas foram calculadas, a saber: Resultado, Margem, Receita Produzida, Receita de Incentivos do SUS, Custos por Grupo de Faturamento, Tempo de Uso da Sala Cirúrgica, Paciente Dia, dentre outros.

\section{Etapa 2: Criação de Dashboards}

Desenvolvido insights de forma que o usuário consiga navegar pelos dados ampliando o nível de detalhamento da informação (drilldown), chegando ao menor grão do cômputo do paciente e item de faturamento. Uma série de filtros também foram adicionados para apoio às análises (Figura 4).

Etapa 3: Aplicação de Algoritmos de Dados

Nessa etapa foram identificadas as variáveis que compõem o resultado assistencial, aplicando algoritmos estatísticos para

Figura 4. Tela do Dashboard Analytics do resultado assistencial.

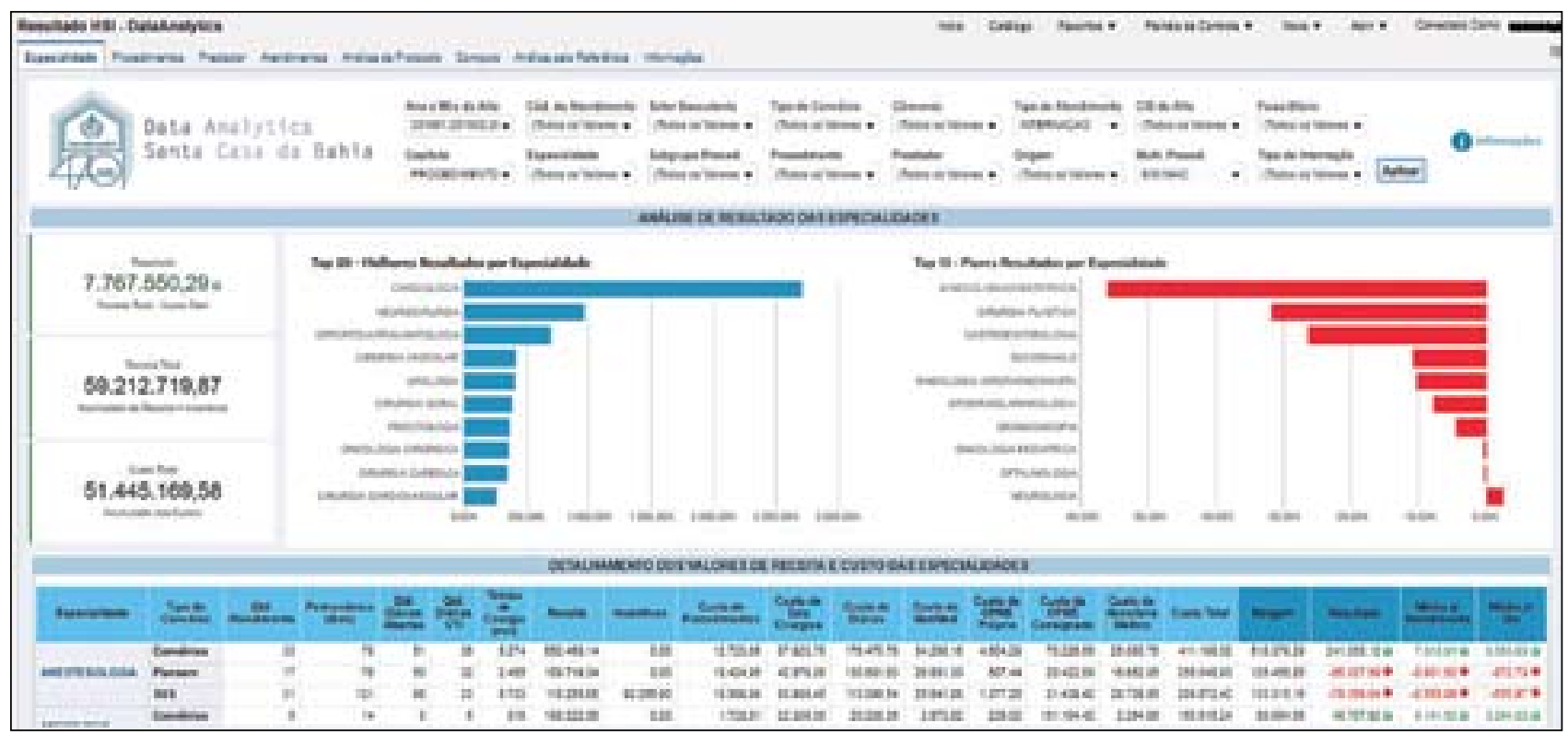


apresentação dos valores de referência para cada uma das variáveis. Os critérios utilizados foram procedimentos cirúrgicos eletivos, não associados, com alta no ano de 2018.

Com base nesse trabalho inicial, oportunidades de análises e aplicação de modelos preditivos estão sendo estudadas, bem como oportunidades em melhorias de processo e registro dos dados, para garantia de uma melhor qualidade do dado.

Outra ação já iniciada trata-se da avaliação de protocolos por procedimento cirúrgico, garantindo otimização dos custos e padronização dos itens com maior probabilidade de ocorrência.

A necessidade de garantir dados que suportem a estratégia, estejam disponíveis, sejam precisos, seguros e acessíveis, torna-se latente nesse contexto e precisam ser tratados a partir de um modelo de governança de dados.

\section{Governança de Dados}

Pesquisas do Gartner publicadas em 2017 e apresentadas em sua conferência de Data \& Analytics, realizada no Brasil, em 2018, apontam que, à medida em que os dados em saúde apresentam maior diversidade e complexidade, observa-se a necessidade de trazer a governança da informação como centro de uma arquitetura mais distribuída, assegurando aspectos de qualidade do dado e melhor entendimento de como o dado pode ser usado, citado na introdução deste artigo como Data Literacy.

Referência mundial em pesquisas ligadas à governança e gestão de dados, a DAMA Data Management Association - desenvolveu o Guia para o Corpo de Conhecimento em Gestão de Dados, conhecido como DMBOK (Data Management Body of Knowledge), que aborda melhores práticas nas diversas áreas de interesse em gestão de dados. O DMBOK apresenta um framework funcional em governança de dados, distribuídos em dez funções distintas, em que cada aspecto da gestão de dados é tratado em grupos de atividades, chamados elementos de ambiente, que, por sua vez, possui escopo bem definido de ações e direcionamentos. Dessa maneira, o DMBOK apresenta um guia de governança e gestão de dados praticado nas organizações em seus mais variados contextos, e que serve como base para as orientações de governança de dados da SCBA.

Desta forma, os domínios de dados mapeados no ambiente de Business Intelligence (BI) e Analytics da SCBA estão orientados pelas melhores práticas da função de Gestão de BI e Data Warehose (DW) da DAMA.

Considerando o projeto de eficiência operacional, a governança está sendo aplicada à medida que dados que geram impacto nos direcionamentos do resultado estão sendo avaliados, tais como agrupamento de diagnósticos, revisão de cadastros de especialidades médicas, dentre outros.

\section{Considerações Finais}

Diante do que foi apresentado, a SCBA e o Hospital Santa Izabel destacam-se no cenário de inovação e transformação digital em saúde com ações que acompanham as tendências tecnológicas de mercado e ciência. Utilizar o dado como direcionador estratégico demonstra tal sinergia, ao passo em que a busca pela maturidade na cultura analítica torna-se fundamental.

Apostar na descentralização do uso da informação e na alfabetização do dado (Data Literacy) são ações que estão em curso e que corroboram com a consolidação desta cultura.

\section{Próximos Passos}

Diversas oportunidades de avanços no projeto de eficiência operacional estão 
sendo consideradas, envolvendo melhorias nas classificações dos procedimentos e no diagnóstico do atendimento. A identificação e padronização dos procedimentos estão sendo avaliadas com relação aos diversos modelos de contratação das operadoras de saúde. Dados clínicos serão incorporados às análises para o desenvolvimento de modelos preditivos e descoberta de novas variáveis. O impacto no uso do Analytics traz mudanças na mentalidade de gestão, permitindo um acompanhamento integrado no que tange à disponibilização dos serviços hospitalares, sem descaracterizar a centralização no cuidado e atenção ao paciente.

\section{Referências}

1. DAMA DMBOK - Guia para o corpo de conhecimento em gerenciamento de dados. $1^{\mathrm{a}}$ Edição, 2012.

2. Craft L. Use the three rings of information governance for classifying healthcare data. Gartner. 2017.
3. Barbieiri C. Governança e gestão estratégica de dados - novas fronteiras, fumsoft. Sociedade Mineira de Software. 2014.

4. Judah S. The state of data and analytics governance: Align risks, optimization and data value iniciatives. Gartner. 2018.

5. Logan VA. Becoming data driven: How to define, develop and deploy data literacy. Gartner. 2018.

6. Kronz A, Schlegel K, Howson C. How to balance control and agility in your self-service analytics. Gartner. 2019.

\section{Notas}

O Grupo Gartner é uma instituição internacional de aconselhamento e pesquisas em tecnologia, que atua de maneira imparcial e com foco em soluções assertivas, baseado nas tendências do mercado tecnológico.

Qmentum - Programa de Acreditação Internacional que orienta e monitora padrões de alta performance em qualidade e segurança na área de saúde.

HIMSS - Healthcare Information and Management Systems Society, que objetiva estimular o uso da tecnologia da informação pelo setor de Saúde. 